\title{
Postoperative time course of INR values in laparoscopic and open liver surgery: a retrospective analysis.
}

\author{
E. Annaert, J. Van Limmen, P. Wyffels, R. Troisi, L. De Baerdemaeker, S. De Hert
}

Department of Anesthesiology and Perioperative Medicine, University Hospital Ghent, Belgium

\section{Background}

- Safe use of epidural analgesia in liver resections.

- Evaluate effect of different surgical approaches for liver resections on INR values in the perioperative period.

\section{Materials and methods}

- Data collection of all liver surgery patients in UZ Gent between 2010 and 2016.

- Data processing as outlined in flowchart.

- INR measurements recorded preoperatively and up to 4th day postoperatively.

- Friedman test, mixed ANOVA and pairwise comparisons for statistical analysis.

Flowchart data processing

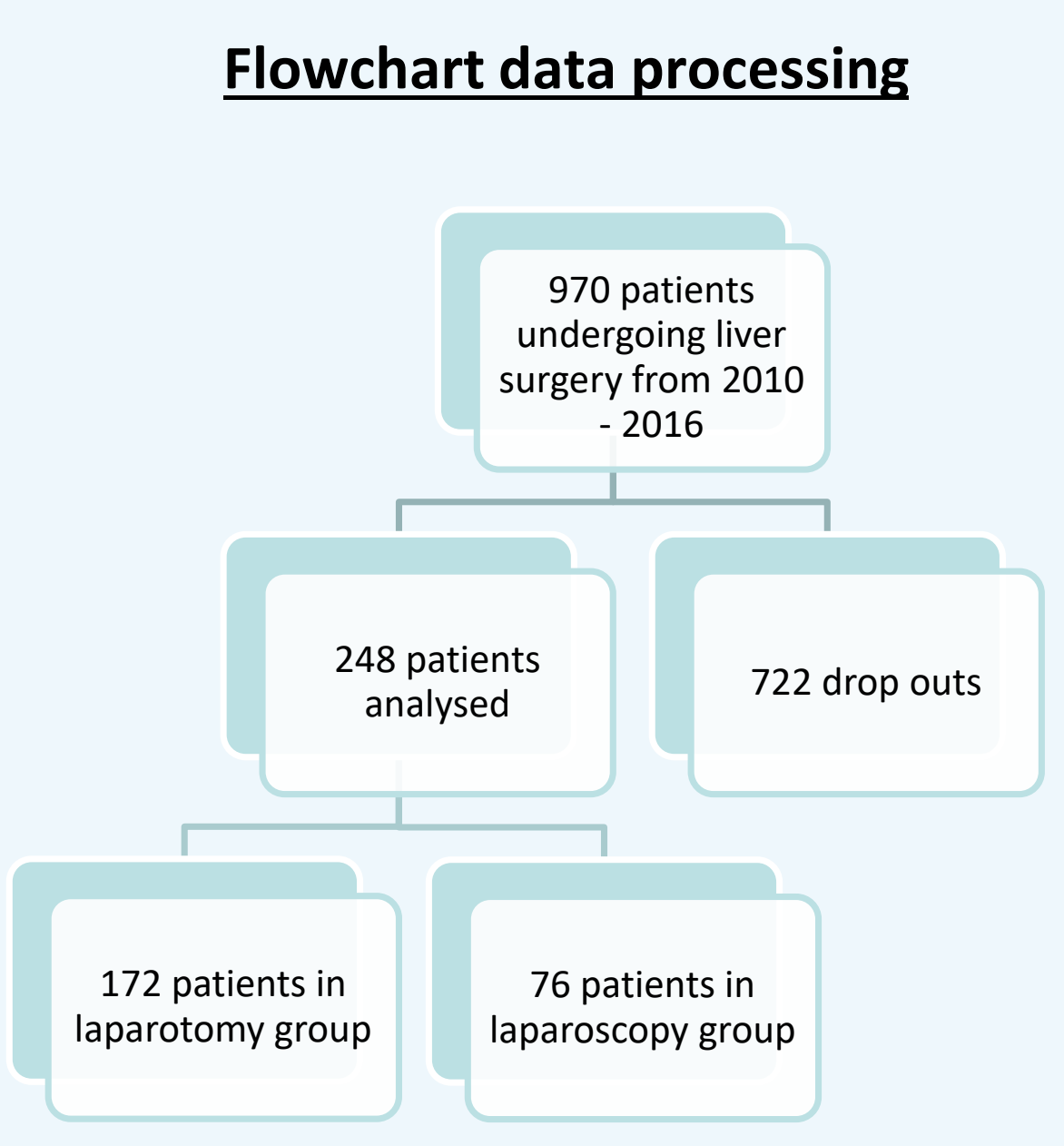

\section{Results}

- For total group of patients analysed, INR values showed significant increase post-operatively.

- Peak INR on post-operative day 2.

- Overall INR values were significantly higher within laparotomy group.

- Pattern of INR changes did not differ significantly between both groups

\section{Conclusion}

In liver resection:

- INR changes postoperatively with peak on postoperative day 2

- Surgical approach does not determine pattern of postoperative INR changes 